\title{
Early Acquisition of Gelatinolytic Activity in Carcinogenesis of the Uterine Cervix
}

Rie Minami, M.D., Hajime Tsunoda, M.D., Tatsuo Iijima, M.D., Hiroyuki Yoshikawa, M.D., Ryoichi Nemori, M.S., Masayuki Noguchi, M.D.

Department of Pathology, Institute of Basic Medical Sciences (RM, TI, MN) and Department of Obstetrics and Gynecology, Institute of Clinical Medical Sciences (RM, HT, HY), University of Tsukuba, Ibaraki; and Fuji Photo Film Co, Ltd, Ashigara Research Laboratories, Kanagawa (RN), Japan

Film in situ zymography is a newly developed technique for detecting in situ gelatinolytic activity. Using the film in situ zymography method to stamp preparations, we evaluated the gelatinolytic activity in early stage cervical neoplasia and invasive squamous cell carcinoma. To determine the sensitivity of film in situ zymography for detecting gelatinase expression, slides made from stamps of 50 specimens resected from the uterine cervix, including early invasive carcinoma (FIGO Ial and Ibl) and cervical intraepithelial neoplasia (CIN) were examined by film in situ zymography. The specimens were also examined immunohistochemically with regard to mitotic activity and gelatinase expression. Gelatinolytic activity was subdivided into two patterns, the homogeneous pattern and the heterogeneous pattern. The homogeneous pattern consisted of circumscribed areas around atypical cell clusters; these areas were composed of homogenously digested full-thickness collagen, whereas the heterogeneous pattern consisted of spottily digested areas of superficial collagen around atypical cell clusters. All invasive carcinomas (8/8 cases) and carcinoma in situ (14/14 cases) were positive for gelatinolytic activity, and $33.3 \%(5 / 15$ cases) of the specimens of CIN-1, CIN-2, and severe dysplasia were also positive. All invasive carcinomas and 6 of 14 carcinoma in situ (43\%) showed homogenous pattern, and the other positive specimens showed heterogenous pattern. The MIB-1 index was $33.8 \%$ in invasive carcinoma, increasing stepwise from dysplasia to carci-

\footnotetext{
Copyright () 2003 by The United States and Canadian Academy of Pathology, Inc.

VOL. 16, NO. 11, P. 1164, 2003 Printed in the U.S.A.

Date of acceptance: July 18, 2003.

This work was supported in part by a Grant-in-Aid (13670162) for Cancer Research from the Ministry of Education, Culture, Sports, Science, and Technology of Japan.

Address reprint requests to: Masayuki Noguchi, M.D., Department of Pathology, Institute of Basic Medical Sciences, University of Tsukuba, Tennodai 1-1-1, Tsukuba-shi, Ibaraki, 305-8575, Japan; fax: +81-298-533150; e-mail: nmasayuk@md.tsukuba.ac.jp.

DOI: 10.1097/01.MP.0000094089.33691.60
}

noma. Matrix metalloproteinase-2 immunostaining was positive in 4 of 8 cases of invasive carcinoma and generally stained the stromal area around the tumor nest. These results indicated that matrix metalloproteinases are functionally activated even in carcinoma in situ and in cervical intraepithelial neoplasia of the uterine cervix not showing invasive growth histologically. Film in situ zymography can detect with sensitivity the invasive potential of carcinoma in situ and cervical intraepithelial neoplasia. An analysis combining cytological examination and film in situ zymography is a potentially useful tool for estimating invasive activity.

KEY WORDS: Dysplasia, Film in situ zymography (FIZ), Matrix metalloproteinase-2, Uterine cervical cancer.

Mod Pathol 2003;16(11):1164-1170

Squamous cell carcinoma of the uterine cervix is a common female genital neoplasm, and because of screening and early detection of premalignant lesions, the incidence of this carcinoma has been decreasing. On the other hand, the occurrence of the disease in young patients has increased recently because of changes in sexual habits, and some of these cases may need to be followed for a long time without radical surgery. To control this carcinoma over a long time period, it is important to estimate risks of recurrence and metastatic and invasive potential, even in premalignant and early-stage lesions.

Matrix metalloproteinases, a family of zinc- and calcium-dependent enzymes, have the ability to degrade components of the extracellular matrix, such as collagen, gelatin, fibronectin, laminin, and proteoglycan. They are considered to be important key enzymes in the initiation of invasion and metastasis (1-3). Expression of matrix metalloproteinases has recently been reported in many kinds of carcinomas, including gynecologic ones (4-12). 
Expression of matrix metalloproteinases in carcinoma has been studied mainly by immunohistochemical staining, in situ hybridization, and zymography (8-13). Although these methods allow us to determine whether or not these gelatinolytic enzymes are expressed in the target tissue, they do not enable us to localize the activity of the matrix metalloproteinases. Film in situ zymography (FIZ) is a newly developed technique that can easily detect in situ gelatinolytic activity, and recent studies have demonstrated that the FIZ technique can detect active expression of matrix metalloproteinases in various kinds of carcinoma tissue $(5,14-16)$.

We used FIZ to determine the gelatinolytic activity in stamp preparations made from material resected from the uterine cervix. We also examined the in situ activity of matrix metalloproteinases in early stage cervical neoplasia and invasive squamous carcinoma.

\section{MATERIALS AND METHODS}

\section{Samples}

Uterine cervix material was obtained from 50 women who underwent conization (Elecrosurgical Unit Model MGI, Honest Medical Co., Tokyo, Japan) at the Department of Gynecology at the University Hospital of Tsukuba and at the Tsukuba Medical Center Hospital, between July 2000 and August 2001. The patients' ages ranged from 18 to 56 years (average, 38 y). After gently wiping the blood and cervical mucus from the specimens, the conization samples were cut in the area that looked most intensively affected on macroscopic examination, and the cut surface was stamped onto a film with a cross-linked gelatin layer. Desquamated cells and tiny tissue fragments on the cut surface-including atypical cells and tumor cell clusters-attached themselves to the film, remaining in their relative places. The basic scheme of the study is summarized in Figure 1.

After stamping had been performed, the remaining material was fixed in $10 \%$ buffered formalin and embedded in paraffin. Histological sections of the cut surface were stained with hematoxylin and eosin (WAKO Pure Chemical Industries, Ltd., Osaka, Japan). The histological diagnosis was made by two pathologists (TI and $\mathrm{MN}$ ) according to the WHO classification. We further subdivided cervical intraepithelial neoplasia (CIN) Grade 3 into carcinoma in situ and severe dysplasia. The 50 sections were diagnosed thus: invasive squamous cell carcinoma ( 5 cases of FIGO Ial and 3 cases of FIGO Ib1), carcinoma in situ (14 cases), severe dysplasia (5 cases), CIN-2 (5 cases), CIN-1 (5 cases), and nontumorous lesion (13 cases). Serial sections of the cut

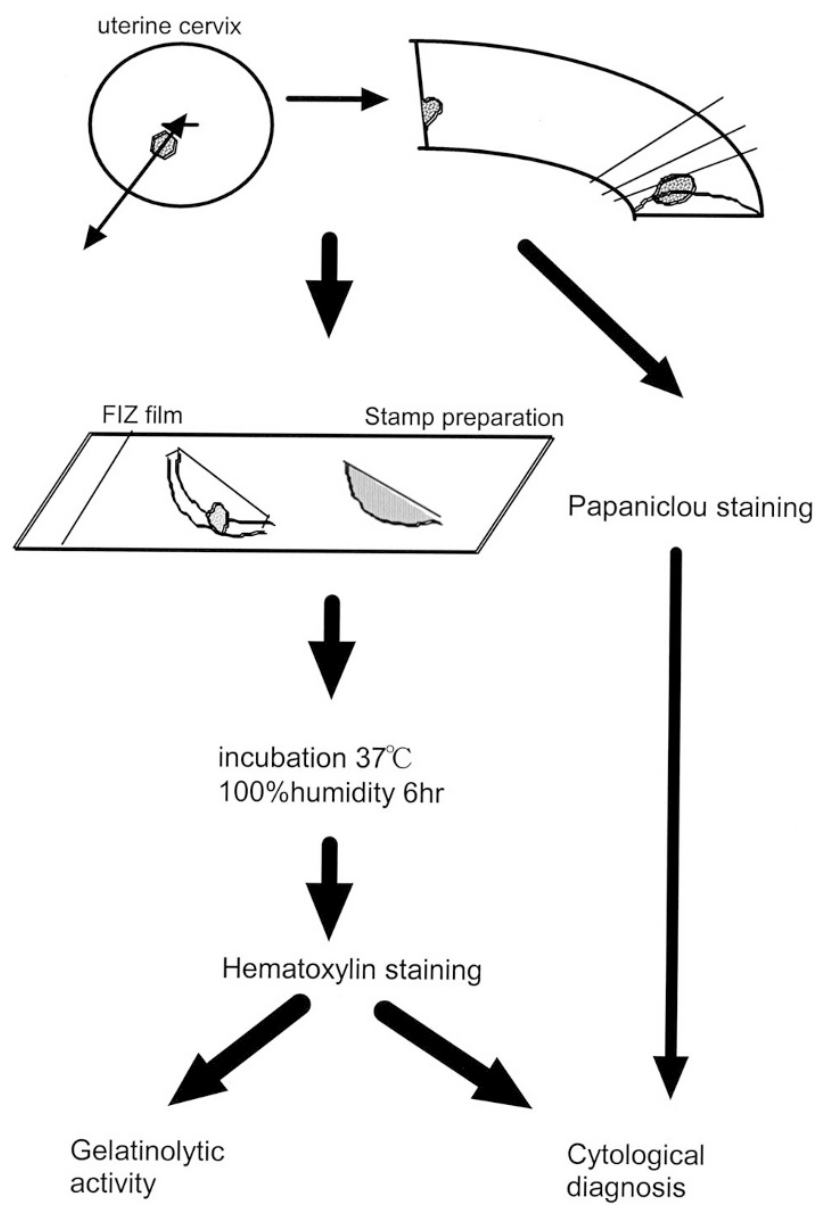

FIGURE 1. Method of film in situ zymography.

surface were prepared for immunostaining for MIB-1 and matrix metalloproteinase-2.

\section{Film In Situ Zymography}

FIZ is a newly developed technique for detecting gelatinolytic activity in situ. The film used in this study has a cross-linked gelatin layer containing dye emulsion coated on a polyethylene terephthalate base film. The gelatin layer is $7 \mu \mathrm{m}$ thick. The emulsion has no effect on the property of gelatin as a protease substrate but adds a pink color to the layer. The stamped film was incubated at $37^{\circ} \mathrm{C}$ for 6 hours in a chamber at $100 \%$ humidity. After the incubation, the film was stained with hematoxylin (WAKO Pure Chemical Industries, Ltd.). The gelatin on the film appears pale pink because it contains dye emulsion, and gelatinolytic activity can therefore be detected by the presence of decolorized areas. Positive findings of gelatinolytic activity were subdivided into two patterns, the homogeneous pattern and the heterogeneous pattern (Figs. 2, 3). The homogeneous pattern indicates strong gelatinolysis, with homogeneous and zonal digestion of circumscribed areas around atypical cell clusters. The heterogeneous pattern indicates weak gelati- 

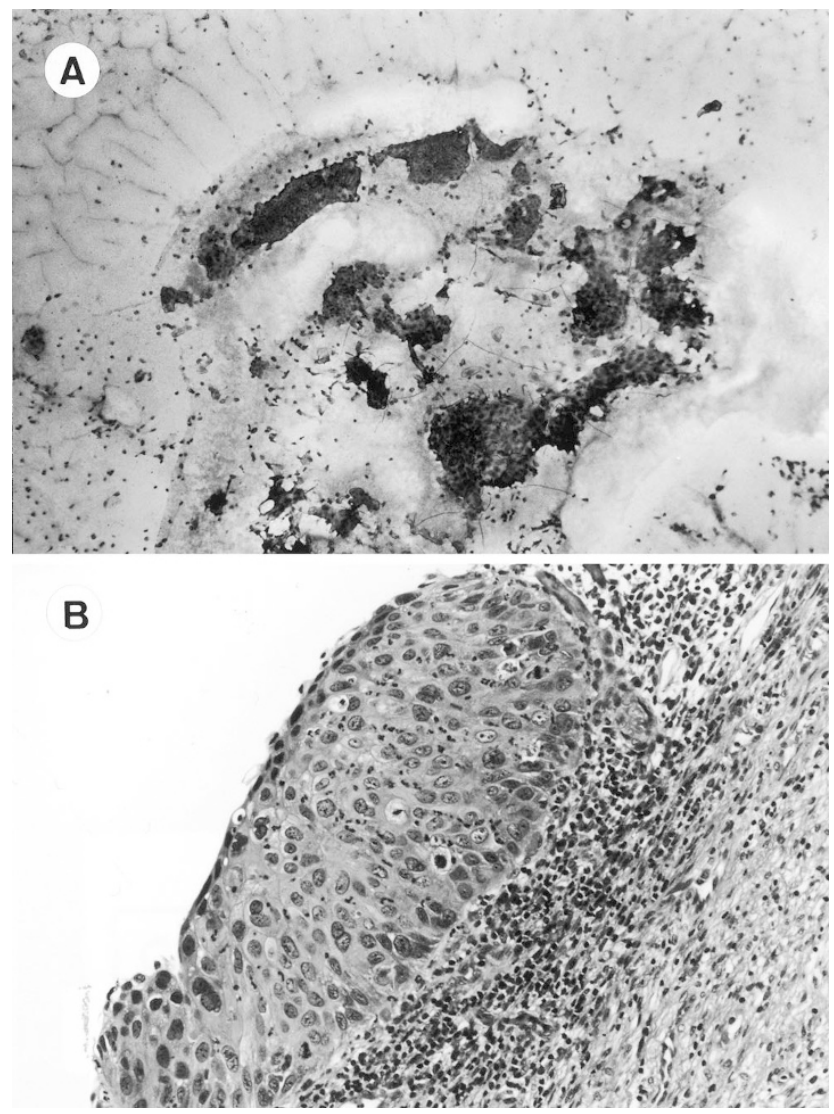

FIGURE 2. Gelatinolytic activity on film in situ zymography: heterogenous pattern. Stamp preparation of severe dysplasia specimens were subjected to film in situ zymography (A) or H \& E staining (B). Heterogenous pattern in film in situ zymography (A) and $\mathrm{H} \& \mathrm{E}$ staining of the serial section (B) (A: original magnification, $40 \times, \mathbf{B}$ : original magnification, $100 \times$ ) Note the different gelatinolytic patterns around the atypical cell cluster. The heterogenous pattern shows paler, decolored background.

nolysis, with spotty and scattered digestion around atypical cell clusters.

The specificity of FIZ was confirmed by treating the film with 1,10-phenanthroline at $100 \mathrm{mmol} / \mathrm{L}$, which suppresses matrix metalloproteinase activity (16; Fig. 4). For the confirmation of FIZ specificity, the levels of active matrix metalloproteinase- 2 and matrix metalloproteinase- 9 were also examined by gelatin zymography (data not shown).

\section{Immunohistochemical Analysis}

To characterize the proliferative activity of the lesions and examine the expression of matrix metalloproteinase-2 protein, Ki-67 and matrix metalloproteinase-2 were immunostained on formalin-fixed, paraffin-embedded tissues by the streptavidin-biotin-peroxidase method (13, 17-19; Fig. 5). We used mouse monoclonal antibody MIB1 (Immunotech Co., Marseilles, France) at a 1:50 dilution to detect Ki67, and mouse monoclonal antibody against matrix metalloproteinase-2 (Fuji Fine chemical, Toyama, Japan; Clone F-68) at a 1:250
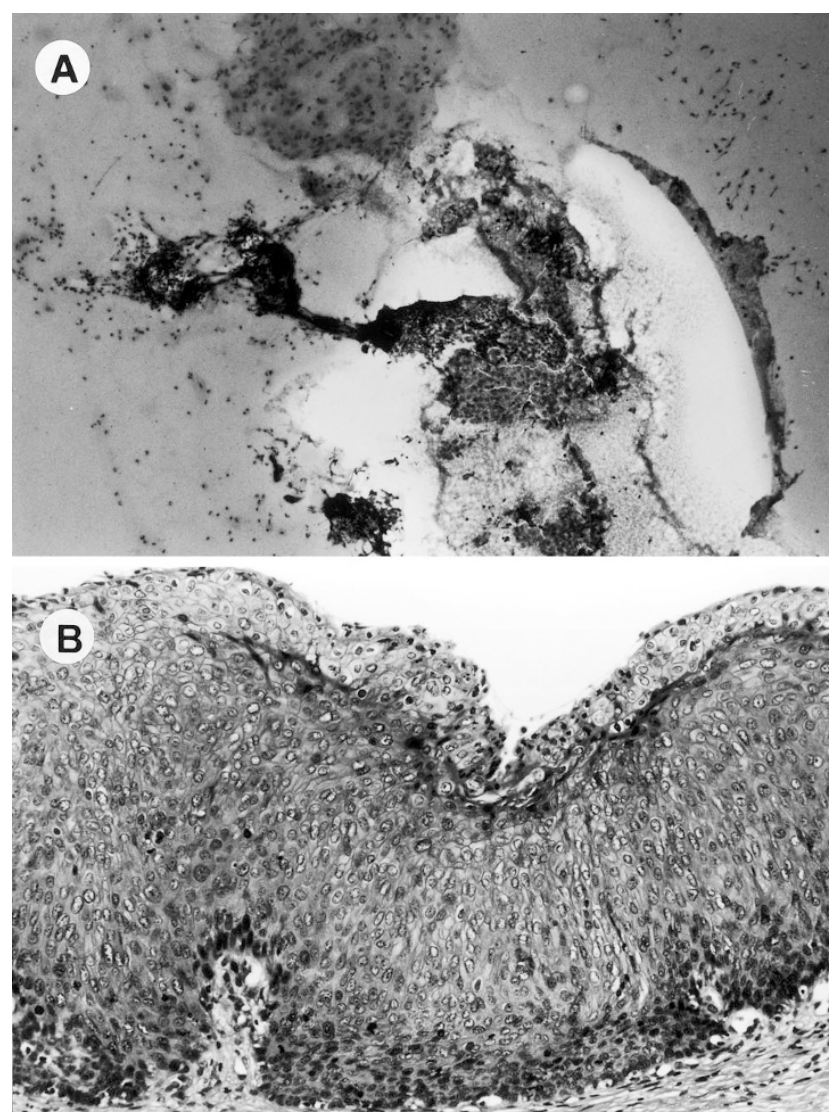

FIGURE 3. Gelatinolytic activity on film in situ zymography: homogenous pattern. Stamp preparation of carcinoma in situ specimens were subjected to film in situ zymography (A) or $\mathrm{H}$ \& $\mathrm{E}$ staining (B). (A: original magnification, $40 \times$, B: original magnification, $100 \times)$. Notice the homogenous pattern with coronal decolored background around the cancer nest. Normal squamous cells were also stamped on the upper part of the film, and no decolored patterns were seen.

dilution. After heat treatment, nonspecific IgG binding and endogenous peroxidase activity were suppressed with $5 \%$ normal bovine serum and $0.3 \%$ hydrogen peroxidase, respectively. For calculation of the MIB-1 index (average number of stained cells per 100 cells counted), stained cells were counted in three different random areas.

\section{Statistical Analysis}

Fisher's exact test and Student's $t$ test were used for the statistical analysis.

\section{RESULTS}

\section{Film In Situ Zymography}

Representative results of the homogeneous pattern and the heterogeneous pattern activity are shown in Figures 2 and 3, and results of FIZ and the immunohistchemistry are summarized in Table 1. All of the cytological diagnoses were histologically confirmed. Eight of 8 invasive carcinoma (100\%) 


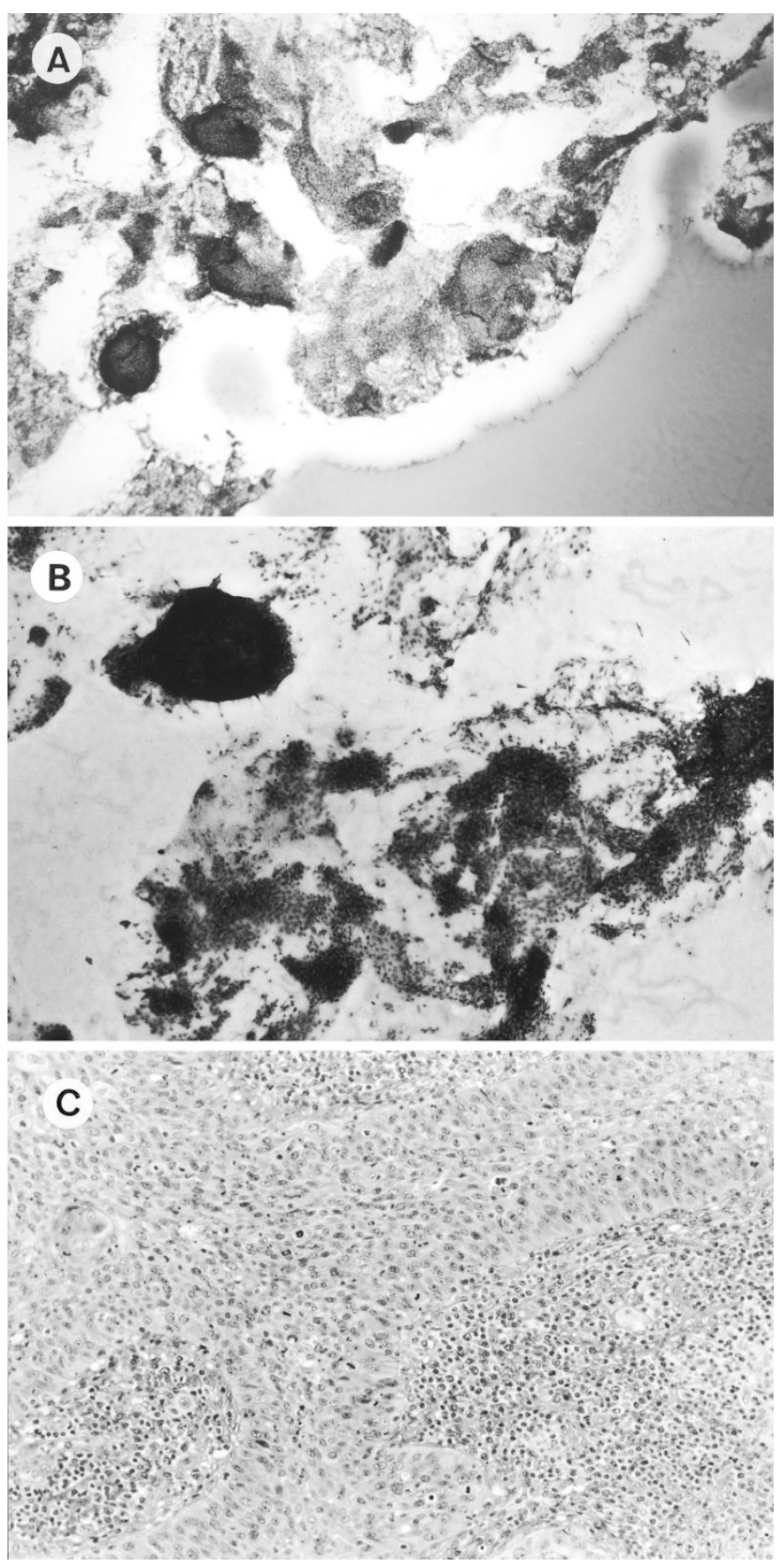

FIGURE 4. Inhibition of in situ gelatinolytic activity by 1.10 phenanthroline and detection of matrix metalloproteinase expression by film in situ zymography. The cancer cell clusters of invasive cervical cancer shows the homogenous gelatinolytic activity (A) and no gelatinolysis on the FIZ film treated by matrix metalloproteinase inhibitor, 1.10-phenanphenanthroline (B). (A, B: original magnification, $40 \times$; C: hematoxylin-eosin staining, original magnification, $40 \times$ )

and 14 of 14 carcinoma in situ (100\%) were positive on FIZ. Five of 15 dysplastic lesions (CIN-1, CIN-2, and severe dysplasia; $33 \%$ ) and 3 of 13 nontumorous lesions $(23 \%)$ were also positive for FIZ. There were significant differences between the positive scores in carcinoma and CINs and nontumorous lesions $(P<.01)$. Of the dysplastic lesions, 5 of 10 CIN-2 and severe dysplasia were positive for FIZ $(50 \%)$, but no CIN-1 (0 of 5 ) showed a positive reaction.
The pattern of positive reactions for FIZ differed according to the pathological diagnosis. Eight of 8 invasive carcinoma (100\%) and 6 of 14 carcinoma in situ $(43 \%)$ showed the homogeneous pattern, and the other positive specimens showed the heterogeneous pattern. These results indicate that almost half of the carcinoma in situ $(43 \%)$ showed the same homogeneous pattern as invasive carcinoma. The heterogeneous pattern was observed in 13 specimens. Even in nontumorous epithelium, the heterogeneous pattern in three specimens was detected in areas in which large numbers of inflammatory cells had accumulated. The gelatinolytic activity was seen to increase according to the malignant progression of the lesions.

\section{Immunohistochemical Analysis}

The results of the immunohistochemical analyses for matrix metalloproteinase-2 and MIB-1 are summarized also in Table 1. MMP-2-positive cells were detected in 4 of 8 invasive carcinoma (50\%; Fig. 5) and none of the other lesions. Matrix metalloproteinase- 2 was mainly expressed in the stromal area just around the cancer nest, and the cancer cells were not stained. All cases positive for matrix metalloproteinase-2 immunostaining were cases with the homogeneous patterns in FIZ. In the positive cases, positive staining for matrix metalloproteinase-2 appeared more strongly at the side nearer to the cancer nest than it did at the distant side. The density of the stained fibroblast around the cancer nests was higher than that of those distant from the nest. The MIB-1 indexes were 7.0 in CIN1 and CIN2, 17.6 in severe dysplasia and carcinoma in situ, and 33.8 in invasive carcinoma. The index increased gradually in a stepwise progression from CIN to invasive carcinoma. The mean MIB-1 index was 39.1 in the 4 matrix metalloproteinase-2-positive cases and 7.8 in the 46 matrix metalloproteinase-2-negative cases.

\section{DISCUSSION}

Recently, FIZ has been reported to be an easy and useful technique for detecting matrix metalloproteinase activity in various types of carcinomas. Several other methods, such as zymography and Northern blot hybridization of matrix metalloproteinases, can be used to examine the function and expression of matrix metalloproteinases in tissues. However, these methods cannot give exact information on the types of cells that express matrix metalloproteinases. In situ hybridization of matrix metalloproteinases is another useful way of detecting expression of matrix metalloproteinases in tissues and cells, but it does not give any information about the functional activity of matrix metallopro- 

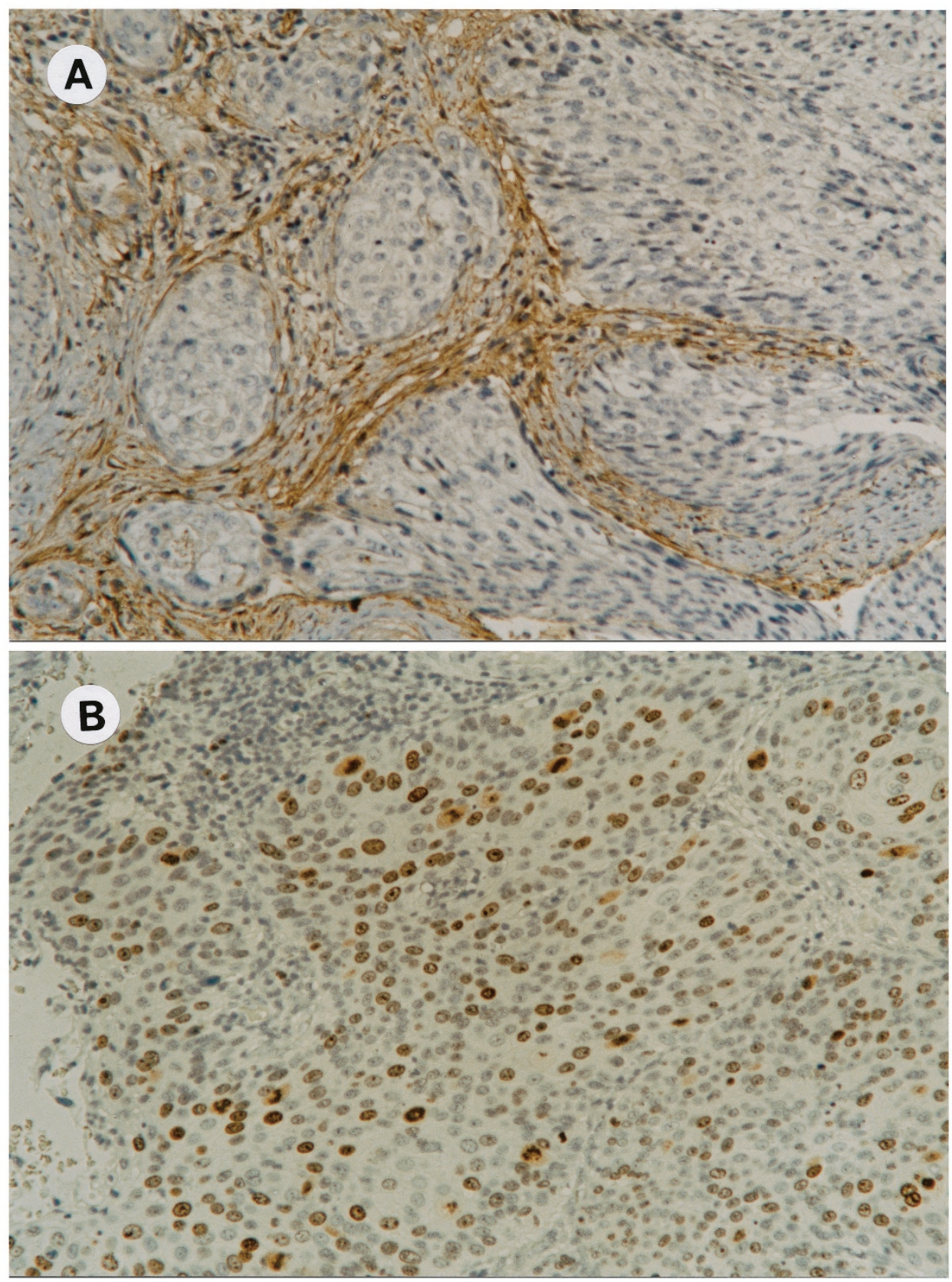

FIGURE 5. Immunohistochemical staining of matrix metalloproteinase-2 and MIB-1 in invasive cervical carcinoma. (A, B: original magnification, $100 \times$ ). Paraffin sections were immunostained with monoclonal antibodies against matrix metalloproteinase-2 (A) and Ki-67 (B). Note that MIB-1 protein was localized in the nuclei of the nonkeratinizing cancer cells, whereas matrix metalloproteinase-2 protein was observed diffusely in surrounding stromal tissues. This case showed the homogenous pattern in film in situ zymography.

teinases. Therefore, FIZ is a very useful method of detecting the activity of matrix metalloproteinases in a short time but also of identifying the types of cells that express activated matrix metalloproteinases. To apply FIZ to cytologic specimens, we chose stamp specimens of the uterine cervix for the following reasons: (1) the sampling procedure is very simple and easy; (2) the cytological and morphological criteria of the dysplastic cells and cancer cells have been established; and (3) all tissue samples resected can be subjected to routine histolog- ical diagnosis, and the results from the cytological specimens can be compared with those from the histological specimens. Our findings can be put easily to practical use for estimating the malignancy of cervical specimens.

Matrix metalloproteinases are involved in not only cancer invasion but also in extracellular matrix turnover activities such as degradation, inflammation, tissue remodeling, and differentiation. In particular, it has been reported that matrix metalloproteinase-9 is induced in chronic and se- 
TABLE 1. Result of Film In Situ Zymography and Immunohistochemistrical Result of Matrix Metalloproteinase-2 and

\begin{tabular}{lccccc}
\hline & Number of cases & Number of HEP (\%) & Number of HOP (\%) & MMP-2 & MIB-1 Index \\
\hline Invasive cancer & 8 & $0(0)$ & $8(100)$ & $4 / 8$ & 33.8 \\
CIN 3 & & & $6(43)$ & $0 / 14$ & 17.6 \\
CIS & 14 & $8(57)$ & $0(0)$ & $0 / 5$ & 7.0 \\
SD & 5 & $3(40)$ & $0(0)$ & $0 / 5$ & 0.9 \\
CIN 2 & 5 & $0(0)$ & $0(0)$ & $0 / 5$ & 0 \\
CIN 1 & 5 & $3(23)$ & $0 / 13$ & 1.0 \\
Nontumorous lesion & 13 & (3) & & \\
\hline
\end{tabular}

CIN, cervical intraepithelial neoplasia; CIS, Carcinoma in situ; SD, severe dysplasia; HEP, heterogenous pattern; HOP, homogenous pattern; MMP-2, matrix metaloproteinase-2; MIB-1 index, average number of the stained cells/100 counted cell.

vere inflammation and might play an important role in tissue remodeling (20-22). These reports suggest that cases that show chronic inflammation also have a positive reaction to matrix metalloproteinase-9 expression. Because the conization materials examined in this study usually included inflammatory changes, we suspect that the gelatinolytic activity in the three cases of nontumorous lesions was caused by matrix metalloproteinase-9. Histologically, these heterogeneous pattern-positive nontumorous cases showed senile colpitis and bacterial vaginalis. Reports of the expression of matrix metalloproteinases in atypical epithelial cells of preinvasive lesions (CIN) have been inconsistent. For example, Davidson et al. (13) indicated that an intense signal of matrix metalloproteinase-2 mRNA was uniformly absent from CIN, but Mattila et al. (11) demonstrated a positive immunohistochemical reaction against matrix metalloproteinase-2 in CIN lesions. In this study, we demonstrated that not only invasive carcinoma, but also carcinoma in situ and dysplastic lesions, showed gelatinolytic activity. It is additionally very interesting that 6 of 14 specimens of carcinoma in situ (43\%) showed high gelatinase activity in a homogeneous pattern, mimicking the eight invasive carcinomas. Although the specific type of matrix metalloproteinase that is responsible for the high gelatinolytic activity cannot be confirmed, these findings might indicate that some carcinoma in situ specimens have latent invasive characteristics similar to those of invasive carcinoma. We reviewed the morphological findings of carcinoma in situ, but these latent invasive carcinoma in situ cannot be discriminated from real carcinoma in situ by cytological and histological findings. Furthermore, the proliferative activity indicated by the MIB-1 index was correlated with the gelatinolytic activity showed in FIZ and the immunohistochemistry.

Immunohistochemical staining of matrix metalloproteinase-2 showed a positive reaction mainly on the cancer stroma in some invasive cases. The sensitivity is lower than that from the FIZ data, and the results did not correlate with the gelatinolytic activity examined by FIZ. However, as matrix metalloproteinase- 2 is activated by the membrane-type matrix metalloproteinase on the tumor surface and secreted around the tumor cells nests, the discrepancy in present study results can be explained by two possibilities: (1) we mainly detected inactivated matrix metalloproteinase-2 protein in the cancer stroma by immnohistochemistry and the results did not correlate with matrix metalloproteinase-2 activity and (2) the gelatinolytic activity detected by FIZ is not due to matrix metalloproteinase-2 but other types of gelatinase. The mechanism of the activation of matrix metalloproteinases in carcinoma in situ needs to be further examined in regard to the roles of tissue inhibitor metalloproteinase- 2 and membrane type 1 matrix metalloproteinase $(2,7-13)$.

In summary, FIZ demonstrated that dysplastic cells of CIN definitively showed gelatinolytic activity but at a lower grade than cells from carcinoma in situ or invasive carcinoma. Furthermore, in some cases of carcinoma in situ, tumor cells appear to have a latently invasive character similar to invasive carcinoma. This FIZ method using cytological specimens is very easy and sensitive, so it will prove very useful for characterizing the invasive potential of CIN and carcinoma in situ. It might be possible to apply this method for taking swab smears in screening for the invasive potential of suspect lesions.

\section{REFERENCES}

1. Coussens LM, Werb Z. Matrix matalloproteinases and the development of cancer. Chem Biol 1996;3:895-904.

2. Aznavoorian S, Murphy AN, Stetler-Stevevson WG, Liotta LA, et al. Molecular aspects of tumor invasion and metastasis. Cancer 1993;71:1368-83.

3. Sato H, Takino T, Okada Y, Cao J, Shinagawa A, Yamamoto E, et al. A matrix metalloproteinases expressioned on the surface of invasive tumor cells. Nature 1994;370:61-5.

4. Zheng ZS, Cohen AM, Guillem JG. Loss of basement membrane type IV collagen is associated with increased expression of metalloproteinases 2 and 9(MMP2and MMP9) during human colorectal tumorigenesis. Carcinogenesis 1999;20:749-55.

5. Nakamura H, Ueno H, Yamashita K, Shimada T, Yamamoto E, Noguchi M, et al. Enhanced production and activation of progelatinase a mediated by membrane type 1 matrix metalloproteinase in human papillary thyroid carcinomas. Cancer Res 1999;59:467-73. 
6. Nakada M, Nakamura H, Ikeda E, Fujimoto N, Yamashita J, Sato $\mathrm{H}$, et al. Expression and tissue localization of membrane type 1,2 and 3 matrix metalloproteinases in human astrocytic tumor. Am J Pathol 1999;154:417-28.

7. Gilles C, Polette M, Piette J, Munaut C, Thompson EW, Birembaut $\mathrm{P}$, et al. High level of MT-MMP expression is associated with invasiveness of cervical cancer cells. Int J Cancer 1996;65:209-13.

8. Garzetti GG, Ciavatti A, Lucarini G, Goteri G, Romanini C, Biagini G. The 72-kDa metalloproteinase immunostaining in cervical carcinoma: relationship with lymph nodal involvement. Gynecol Oncol 1996;60:271-6.

9. Garzetti GG, Ciavatti A, Lucarini G, Goteri G, Nictolis MD, Romanini C, et al. Microinvasive cervical carcinoma and cervical intraepithelial neoplasia: biologic significance and clinical implications of 72-kDa metalloproteinase immunostaining. Gynecol Oncol 1996;61:197-203.

10. Nuovo GJ. In situ detection of PCR-amplified metalloproteinase cDNAs, their inhibitors and human papillomavirus transcripts in cervical carcinoma cell lines. Int J Cancer 1997;71:1056-60.

11. Mattila AT, Sarkkinen AM, Hoytya M, Westerlund A, Puistola U, Hujanen TT. Matrix metalloproteinase 2 immunoreactive protein appears early in cervical epithelial dedifferentiation. Gynecol Oncol 1999;72:306-11.

12. Davidson B, Goldberg I, Kopolovic J, Geva LL, Gotlieb WH, Weis B, et al. Expression of matrix metalloproteinase-9 in squamous cell carcinoma of the uterine cervixclinicopathological study using immunohistochemistry and mRNA in situ hybridization. Gynecol Oncol 1999;72:380-6.

13. Davidson B, Goldberg I, Kopolovic J, Geva LL, Gotlieb WH, Baruch GB, et al. MMP-2 and TIMP-2 expression correlates with poor prognosis in cervical carcinoma-a clinicopatho- logic study using immunohistochemistry and mRNA in situ hybridization. Gynecol Oncol 1999;73:372-82.

14. Abiko Y, Katsuzawa M, Kowashi Y, Kaku T, Tachikawa T. In situ detection of gelatinolytic activity in developing craniofacial tissues. Anat Embryol 1999;200:283-7.

15. Furuya M, Ishikura H, Nemori R, Shibata M, Fujimoto S, Yoshiki T. Clarification of the active gelatinolytic sites in human ovarian neoplasms using in situ zymography. Hum Pathol 2001;32:163-8.

16. Ikeda M, Maekawa R, Tanaka H, Matsumoto M, Takeda $Y$, Tamura Y, et al. Inhibition of gelatinolytic activity in tumor tissues by synthetic matrix metalloproteinase inhibitor: application of film in situ zymography. Clin Cancer Res 2000; 6:3290-6.

17. Nakano T, Oka K. Differential values of Ki-67 index and mitotic index of proliferating cell population. Cancer 1993; 72:2401-8.

18. Suzuki M, Tsukagoshi S, Saga Y, Ohwada M, Sato I. Assessment of proliferation index with MIB-1 as a prognostic factor in radiation therapy for cervical cancer. Gynecol Oncol 2001; 79:300-4.

19. Clarke B, Chetty R. Cell cycle aberrations in the pathogenesis of squamous cell carcinoma of the uterine cervix. Gynecol Oncol 2001;82:238-46.

20. Vaday GG, Schor H, Rahat MA, Lahat N, Ofer L. Transforming growth factor- $\beta$ suppresses tumor necrosis factor- $\alpha$ induced matrix metalloproteinase- 9 expression in monocytes. J Leukoc Biol 2001;69:613-21.

21. Owen CA, Campbell EJ. The cell biology of leukocytemediated proteolysis. J Leukoc Biol 1999;65:137-50.

22. Kanbe N, Tanaka A, Kanbe M, Itakura A, Kurisawa M, Matsuda H. Human mast cell produce matrix metalloproteinase 9. Eur J Immunol 1999;29:2645-9. 